\title{
THE EXISTENCE OF DUAL MODULES
}

\author{
D. D. ANDERSON
}

\begin{abstract}
In this note we show that a Noetherian module has a dual module if and only if it satisfies $A B 5^{*}$. A connection between completeness and $A B 5^{*}$ is also established.
\end{abstract}

In this note we relate completeness, quasi-completeness, the $A B 5^{*}$ condition, and duality. The main result is that a Noetherian $R$-module has a dual module if and only if it satisfies $A B 5^{*}$.

Throughout this note $R$ will denote a commutative ring with identity and all modules will be unitary. The terms local and semilocal will carry the Noetherian hypothesis. We use $L(A)$ or $L_{R}(A)$ to denote the lattice of $R$ submodules of $A$.

An $R$-module $B$ is said to be a dual of an $R$-module $A$ if there exists an order reversing lattice isomorphism $\theta: L(A) \rightarrow L(B)$ satisfying $\theta(J N)=\theta(N): J$ for all $R$-submodules $N$ of $A$ and all ideals $J$ of $R$.

Any $R$-module $A$ satisfies the so-called $A B 5$ condition: for any submodule $B$ and any ascending chain $\left\{B_{\alpha}\right\}$ of submodules of $A, B \cap\left(\cup_{\alpha} B_{\alpha}\right)=$ $\cup_{\alpha}\left(B \cap B_{\alpha}\right)$. $A$ satisfies the dual condition $A B 5^{*}$ if for any submodule $B$ and any descending chain $\left\{B_{\alpha}\right\}$ of submodules, it follows that $B+\left(\cap_{\alpha} B_{\alpha-}\right)=$ $\bigcap_{\alpha}\left(B+B_{\alpha}\right)$. Not every module satisfies $A B 5^{*}$; for example, $Z$, the integers, does not. However, any module having a dual necessarily satisfies $A B 5^{*}$. We show that for Noetherian modules, the converse is also true. We first show that the condition $A B 5^{*}$ is closely related to completeness.

Let $R$ be a semilocal ring with Jacobson radical $J$ and let $A$ be a finitely generated $R$-module. If $A$ is complete in the $J$-adic topology, it is well known [6] that $A$ satisfies the condition

(*) For any descending chain $\left\{B_{n}\right\}_{n=1}^{\infty}$ of submodules of $A$ and any integer $k$, there exists an integer $n(k)$ such that $B_{n(k)} \subseteq\left(\cap_{n=1}^{\infty} B_{n}\right)+J^{k} A$.

A finitely generated module over a semilocal ring will be called quasicomplete if it satisfies $(*)$.

The first theorem relates the concepts of quasi-completeness and $A B 5^{*}$.

THEOREM 1. Let $R$ be a semilocal ring and $A$ a finitely generated $R$-module. Then $A$ satisfies $A B 5^{*}$ if and only if it is quasi-complete.

Proof. Suppose $A$ satisfies $A B 5^{*}$. Let $\left\{B_{n}\right\}_{n=1}^{\infty}$ be a countable descending chain of submodules of $A$ and let $k$ be a fixed integer. Then

$$
J^{k} A+\bigcap_{n=1}^{\infty} B_{n}=\bigcap_{n=1}^{\infty}\left(J^{k} A+B_{n}\right)=J^{k} A+B_{n(k)}
$$

Received by the editors June 5, 1975.

AMS (MOS) subject classifications (1970). Primary 13C05, 13E05, $13 \mathrm{~J} 10$.

Key words and phrases. $A B 5^{*}$, complete module, dual module. 
for some integer $n(k)$ since $A$ satisfies $A B 5^{*}$ and $A / J^{k} A$ is Artinian. Thus $B_{n(k)} \subseteq \cap_{n=1}^{\infty} B_{n}+J^{k} A$, so $A$ is quasi-complete. Conversely, suppose that $A$ is quasi-complete. Let $\left\{B_{n}\right\}_{n=1}^{\infty}$ be a countable descending chain in $A, B$ $=\bigcap_{n=1}^{\infty} B_{n}$, and let $C$ be a submodule of $A$. We show that $C+B$ $=\cap_{n=1}^{\infty}\left(C+B_{n}\right)$. For fixed $k$, by quasi-completeness, there exists an integer $n(k)$ such that $B_{n(k)} \subseteq B+J^{k} A$, and hence $C+B_{n(k)} \subseteq C+B+J^{k} A$. We may assume $n(k) \rightarrow \infty$ as $k \rightarrow \infty$. Hence

$$
\bigcap_{n=1}^{\infty}\left(C+B_{n}\right)=\bigcap_{n=1}^{\infty}\left(C+B_{n(k)}\right) \subseteq \bigcap_{k=1}^{\infty}\left(B+C+J^{k} A\right)=B+C
$$

by the Krull Intersection Theorem. The reverse containment is always true. The result now follows since any chain in $A$ is countable [2].

We next relate completeness and quasi-completeness. Let $R$ be semilocal with Jacobson radical $J$ and let $A$ be a finitely generated $R$-module. $L(A)$, the lattice of submodules of $A$ has a natural metric $d$ defined on it by $d(C, D)$ $=2^{-n}$ if $C+J^{n} A=D+J^{n} A$ but $C+J^{n+1} A \neq D+J^{n+1} A$. The next theorem is due to E. W. Johnson [3].

Theorem 2. Let $R$ be a semilocal ring and $A$ a finitely generated $R$-module. Then the following are equivalent:

(1) the metric $d$ on $L(A)$ is complete,

(2) $A$ is quasi-complete,

(3) the map $L_{R}(A) \rightarrow L_{\hat{R}}(\hat{A})$ given by $N \rightarrow \hat{R} N$ (where `denotes the J-adic completion) is surjective (and hence a lattice isomorphism).

We remark that while any complete module is quasi-complete, a quasicomplete module need not be complete. For example, any $D \vee R$ is quasicomplete. More generally a one-dimensional local domain is quasi-complete if and only if it is analytically irreducible. The ring $k[X, Y]_{(X, Y)}, k$ a field, is not quasi-complete.

The main theorem requires the following

LEMMA. Let $R$ be a Noetherian ring and $A$ a finitely generated $R$-module satisfying $A B 5^{*}$. Then $\operatorname{Supp}(A)$ contains only finitely many maximal elements; actually each $P \in \operatorname{Ass}(A)$ is contained in a unique maximal element of $\operatorname{Supp}(A)$.

Proof. Since $\operatorname{Ass}(A)$ is finite, the second statement implies the first. For $P \in \operatorname{Ass}(A), R / P$ is isomorphic to a submodule of $A$ and hence satisfies $A B 5^{*}$ as an $R$-module and hence as a ring. Thus we are reduced to showing that a Noetherian domain $R$ satisfying $A B 5^{*}$ must be local. Suppose not, say $P$ and $Q$ are distinct maximal ideals in $R$. Now $\left\{P^{n}\right\}_{n=1}^{\infty}$ is a descending chain of ideals in $R$ and $\cap_{n=1}^{\infty}=0$ by the Krull Intersection Theorem. Hence $Q+\cap_{n=1}^{\infty} P^{n}=Q$. However, for every $n, Q+P^{n}=R$, so $\cap_{n=1}^{\infty}\left(Q+P^{n}\right)$ $=R$. Thus $R$ must be local.

Finally, we require the theory of duality between Noetherian and Artinian modules over a complete local (or semilocal) ring given by Matlis [4] and [5]. (Also see [7] for an introduction into injective modules and duality.) Briefly, let $R$ be a complete semilocal ring with Jacobson radical $J$. There is a perfect duality between Noetherian and Artinian $R$-modules given by the functor $\operatorname{Hom}_{R}(-, E(R / J))$ where $E(R / J)$ is the injective envelope of $R / J$. Also for $R$ 
semilocal, but not necessarily complete, and for $A$ an Artinian $\hat{R}$-module, the $R$-submodules and $\hat{R}$-submodules coincide and hence $A$ is also Artinian as an $R$-module.

THEOREM 3. For a finitely generated module $A$ over a Noetherian ring $R$, the following are equivalent:

(1) A has a dual,

(2) A satisfies $A B 5^{*}$

(3) $A$ is quasi-complete as an $\bar{R}=R / \operatorname{ann}(A)$ - module.

Proof. It is clear that (1) implies (2). Suppose $A$ satisfies $A B 5^{*}$. Then $A$ satisfies $A B 5^{*}$ as an $\bar{R}$-module. By the previous lemma, $\bar{R}$ is semilocal. By Theorem $1, A$ is quasi-complete as an $\bar{R}$-module. It remains to show that (3) implies (1). By change of rings, it suffices to show that $A$ has an $\bar{R}$-module dual. Thus we may replace $R$ by $\bar{R}$ and assume that $R$ is semilocal. By Theorem 2 , the map $L_{R}(A) \rightarrow L_{\hat{R}}(\hat{A})$ given by $N \rightarrow \hat{R} N$ is a lattice isomorphism which preserves scalar product (i.e., $\hat{R}(J N)=J(\hat{R} N))$. Now as an $\hat{R}$ module, $\hat{A}$ has a dual, namely, $B=\operatorname{Hom}_{\hat{R}}(\hat{A}, E(\hat{R} / \hat{J}))$. Since $B$ is Artinian as an $\hat{R}$-module, it follows that the $R$-submodules of $B$ coincide with the $\hat{R}$ submodules of $B$. Hence $B$ is actually an $R$-module dual of $A$.

We have shown that a Noetherian module has a dual if and only if it satisfies $A B 5^{*}$. The hypothesis that the module be Noetherian cannot be deleted. Any Artinian module satisfies $A B 5^{*}$; however, it is easily seen that the abelian group $Z_{p^{\infty}}$ ( $p$ a prime) does not have a $Z$-module dual. In fact, a result of Baer [1] states that an abelian group has a dual if and only if it is torsion and every primary component is finitely generated.

\section{REFERENCES}

1. R. Baer, Dualism in abelian groups, Bull. Amer. Math. Soc. 43 (1937), 121-124.

2. H. Bass, Descending chains and the Krull ordinal of commutative Noetherian rings, J. Pure Appl. Algebra 1 (1971), no. 4, 347-360. MR 46 \# 1778.

3. E. W. Johnson, A note on quasi-complete local rings, Colloq. Math. 21 (1970), 197-198. MR $42 \# 262$.

4. E. Matlis, Injective modules over Noetherian rings, Pacific J. Math. 8 (1958), 511-528. MR 20 \#5800.

5. - Modules with descending chain condition, Trans. Amer. Math. Soc. 97 (1960), 495-508. MR 30 \# 122.

6. M. Nagata, Local rings, Interscience Tracts in Pure and Appl. Math., no. 13, Interscience, New York, 1962. MR 27 \#5790.

7. D. W. Sharpe and P. Vamos, Injective modules, Cambridge Univ. Press, Cambridge, 1972.

Department of Mathematics, University of Iowa, Iowa City, Iowa 52242

Current address: Department of Mathematics, Virginia Polytechnic Institute and State University, Blacksburg, Virginia 24061 